\title{
Quantitative evaluation of sense of discrepancy of operation response using event-related potential
}

\author{
Kazutaka UEDA*, Yuki SAKAI* and Hideyoshi YANAGISAWA* \\ *Department of Mechanical Engineering, \\ Graduate School of Engineering, The University of Tokyo \\ 7-3-1 Hongo, Bunkyo-ku, Tokyo, 113-8656 Japan \\ E-mail: ueda@design-i.t.u-tokyo.ac.jp
}

Received: 30 July 2020; Revised: 27 October 2020; Accepted: 4 March 2021

\begin{abstract}
A user operating a mechanical devise receives sensory information (e.g., sounds and vibrations) generated by the mechanical operation in response to the operation input. With the digitization of products, the mechanical operation response is removed as is the associated sensory information, thus there is no confirmation of the completed operation. Adding a digital sound or vibration could solve this problem. The challenge, however, is designing an operation response without inducing any sense of discrepancy. The purpose of this study was to create a quantitative, objective method to evaluate the sense of discrepancy to the operation response and the habituation to the response. We investigated the possibility of using an event-related potential component (P300), believed to reflect attention to stimuli, as a quantitative evaluation method of the sense of discrepancy. Experiment 1: We studied the sense of discrepancy between the type of operation response (sound, vibration, none), and the shutter operation of a mirrorless single-lens camera, using subjective evaluation and the P300. We confirmed that the P300 amplitude corresponded to the degree of the sense of discrepancy. Experiment 2: With the mouse click being the operation sound, the attenuation of the P300 amplitude by the repeated operation was consistent with the subjective habituation to the response. We conclude, based on these results, that the P300 amplitude could help determine the sense of discrepancy to the operation response and the habituation to the response.
\end{abstract}

Keywords : Operation response, Product, Sense of discrepancy, Habituation, Event-related potential, P300

\section{Introduction}

When operating many conventional consumer products, the user perceives sensory information, such as sounds and vibrations, generated by the operation of mechanical mechanisms in response to the operation input. However, due to the recent simplification and digitization of products, the lack of mechanical operation responses has resulted in a concomitant absence of the associated sensory information, causing problems such as the failure to obtain confirmation for completed operations. Taking a camera as an example, when the shutter is released in a conventional single-lens reflex camera, sound and vibration are emitted as the shutter curtain descends. Conversely, mirrorless single-lens digital cameras do not generate sound or vibration. While this problem may be conceivably solved by adding digital sound and vibration to the product, it remains necessary to design a response that does not give users any sense of discrepancy between their action on the device and the resulting operation and that the response is one that is easily habituated to. This study sought to devise a method to quantitatively evaluate the user's sense of discrepancy between the response and product operation, and the habituation time to the response. We examined the possibility of using the P300, which is an event-related brain potential (ERP) component, as a method of quantitative evaluation of the sense of discrepancy. The P300 is a positive component of the electroencephalogram (EEG) that appears predominantly over the parietal cortical area at a latency of approximately $250-600 \mathrm{~ms}$ after stimulus presentation, and is considered to reflect attention to and novelty (e.g., surprise) of stimuli (Gray et al., 2004; Polich, 2007; Yanagisawa et al., 2019). It is thought that when a user feels a sense of discrepancy with the operation response, he or she will pay more attention to the operation response according to the degree of discrepancy, and the P300 amplitude will increase. In a series of studies on brain activity related to habituation to stimuli using the P300, Polich et al. showed that the P300 amplitude was attenuated by 
repeated exposure to the stimuli (Polich, 1989; Lew and Polich, 1993; Polich and McIsaac, 1994; Romero and Polich, 1996; Ravden and Polich, 1998; 1999). In recent years, several studies have also examined the habituation to stimuli using P300 (Pan et al., 2000; Wintink et al., 2001; Kececi et al., 2006; Mars et al., 2008; Fonken et al., 2019; Barry et al., 2020; Ueda et al., 2020). However, they have all investigated habituation to simple or passive stimuli. We have not found a study that has quantitatively assessed the sense of discrepancy to the operation response and the habituation to the response by operating a real product. Therefore, we aimed to test the applicability of P300 as a quantitative evaluation method of the sense of discrepancy and the habituation time of the operation response by operating the real product. In Experiment 1, we investigated the sense of discrepancy between the lack of operation response (sound, vibration) and the shutter operation of a single-lens camera, using subjective evaluation and the P300. In Experiment 2 , we investigated the habituation of P300 amplitude during a repetitive operation using sounds that were easy or difficult to get used to as the operation response. We also measured the subjective evaluation of distractibility to the operation response according to the degree of discrepancy in Experiment 2. We hypothesized that the P300 amplitude would increase with the increasing sense of subjectively-rated discrepancy, and that the attenuation over time of the subjectively-rated distractibility and the P300 amplitude would show the same trend (i.e., similar pattern of habituation).

\section{Experiment 1: Evaluation of the sense of discrepancy of operation response using the P300}

In product design, it is necessary to decide which sensory modality should be used as the product's operation response, considering its usage. If we can evaluate the differences in the sense of discrepancy between different operation response modalities, we can improve product design. In Experiment 1, we tested whether the P300 can quantitatively evaluate the sense of discrepancy of the operation response in a system that simulates product operation. We used a single-lens camera as the product because users have some expectations of the operation response from experience and social convention, and because we could arbitrarily control the operation response of the actual product. Operational responses in single-lens cameras include auditory, tactile, and visual sensory modalities. For instance, the camera's operation response to the user's operation of the release button is often based on the shutter sound as an auditory stimulus, vibration as a tactile stimulus, and flashing lights on the viewfinder and screen as a visual stimulus. There are two possible shooting postures using a single-lens camera: one is to look through the viewfinder, and the other is to take a picture without looking through the viewfinder, with the face away from the camera body. Therefore, in this experiment, we focused on shutter sound and vibration as these are typically perceived, regardless of shooting posture. In this study, we used the oddball paradigm, which measures the brain response to low-frequency stimuli by changing the presentation frequency depending on the stimulus (Polich, 2007). We hypothesized that the sense of discrepancy would be subjectively perceived for low-frequency operation responses, and the intensity of the sense of discrepancy would be reflected in the amplitude of P300.

\subsection{Methods}

\subsubsection{Participants}

We recruited seven right-handed volunteers (five men; age range, 22-26 years) who had neither brain-related disorders nor any other disease. Handedness was measured using the Japanese version of the FLANDERS handedness test (Okubo et al., 2014). None of the participants had professional experience in using the camera. The study protocol was approved by the Ethics Committee of the Graduate School of Engineering, the University of Tokyo. All participants provided written informed consent prior to their participation in this study.

\subsubsection{Apparatus and stimuli}

The experimental setup is illustrated in Fig. 1. When the participant pressed the release button on a mirrorless camera ( $\alpha 7$ Mark II, Sony, Tokyo, Japan), vibration and sound were presented. The input triggered by the button press and the following stimulus presentation were controlled using the stimulus presentation control software (Presentation, Neurobehavioral Systems, Inc., California, U.S.A.). A square wave with a frequency of $320 \mathrm{~Hz}$ and a duration of 120 
ms was used as the vibration stimulus, which was amplified (Power Amplifier Type 2706, Brüel \& Kjær, Nærum, Denmark) and emitted by a linear actuator $(9 \times 10 \times 22.6 \mathrm{~mm})$ placed inside the camera grip (Fig. 2). During the experiment, participants held the camera's grip with their right hand and received a vibration stimulus on the palm of their hand. The shutter sound used in the actual $\alpha 7$ Mark II was used as the auditory stimulus (volume, $74 \mathrm{~dB}$; duration, $120 \mathrm{~ms}$ ). Noise canceling earphones (QuietComfort20, Bose, Massachusetts, U.S.A.) were used to present the shutter sound. Participants wore earmuffs over the headphones to eliminate the influence of the sound of the vibration.

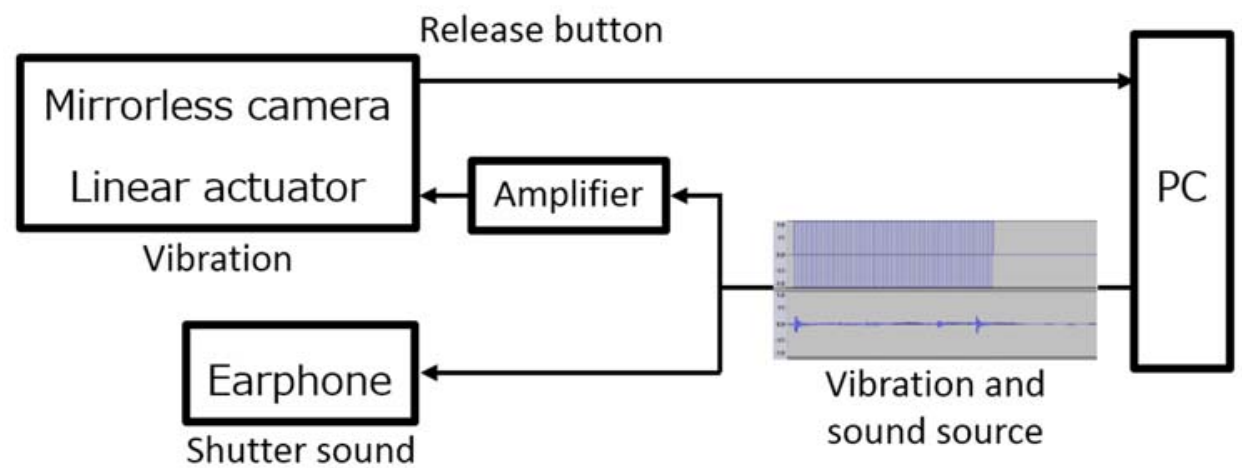

Fig. 1 Outline of experimental setup.

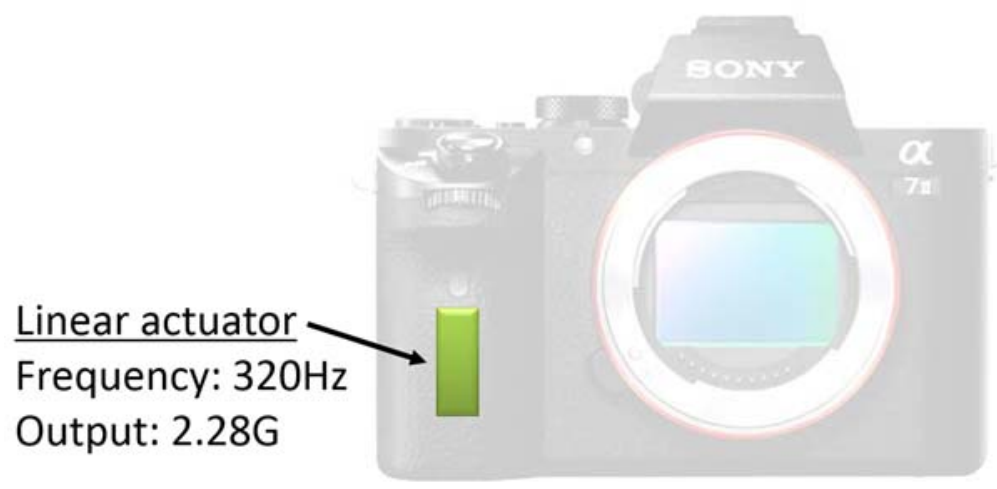

Fig. 2 Position of the linear actuators in the camera.

\subsubsection{Procedure}

After the experiment was explained to the participants, they were instructed to sit comfortably on a chair while conducting the following operation task (Fig. 3). Participants continuously pressed the release button at intervals of 1-2 s. A neutral landscape photograph, which was selected so as not to evoke any emotion, was always presented on the display (MultiSync LCD-PA302W-BK, NEC, Tokyo, Japan). Every time the release button was pressed, one of four types of operation responses was presented in a random order: both the sound and vibration stimuli (audio and tactile condition, 70 trials, high-frequency response), only an auditory stimulus (audio-only condition, 10 trials, low-frequency response), only a vibration stimulus (tactile-only condition, 10 trials, low-frequency response), and no stimuli (none condition, 10 trials, low-frequency response). The participants were asked to rate the intensity of their sense of discrepancy for each condition's operation response on a four-point Likert scale ( 1 = "no discrepancy", $4=$ "high discrepancy") at the end of the operation task. 


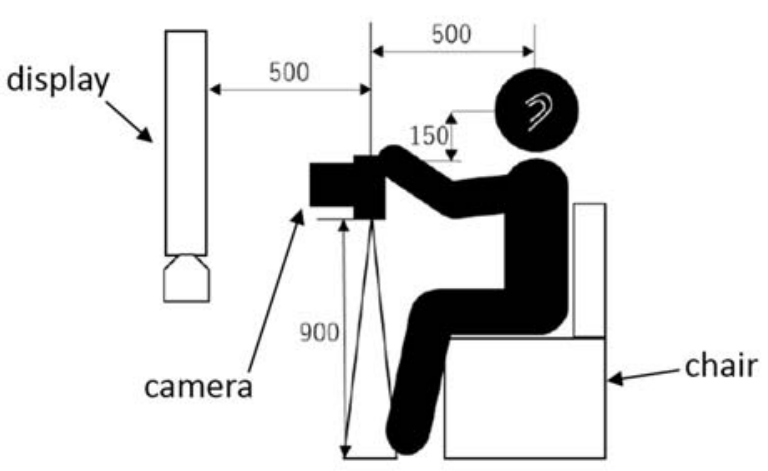

Fig. 3 Outline of experimental setup (unit: $\mathrm{mm}$ ).

\subsubsection{EEG recordings}

The EEG data were recorded during tasks using a digital recorder (Polymate AP1132, TEAC Corporation, Tokyo, Japan) and EEG recording software (AP Monitor, TEAC Corporation, Tokyo, Japan). EEGs were recorded from 30 active electrodes on the scalp positioned according to the international 10-20 system (Fp1/Fp2, F7/F8, F3/F4, Fz, FT7/ FT8, FC3/ FC4, FCz, T7/ T8, C3/ C4, Cz,TP7/TP8, CP3/ CP4, CPz, P7/ P8, P3/ P4, Pz, O1/ O2, and Oz, Fig. 4) (Klem et al., 1999), with an electrode placed on the top of the nose as a reference $(\mathrm{Nz})$. The data were recorded at a sampling frequency of $500 \mathrm{~Hz}$, and the time constant was set to $3 \mathrm{~s}$. All electrode impedances were below $50 \mathrm{k} \Omega$.

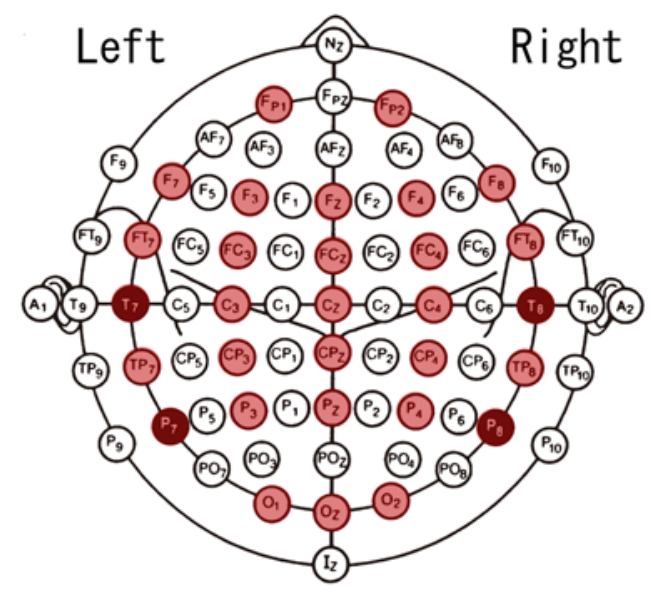

Fig. 4 EEG electrode placement (red circles). This diagram was obtained and modified from Klem et al. (1999).

\subsubsection{EEG data analysis}

The EEG data were exported to EEGLAB14.1.1b (MATLAB toolbox) (Delorme and Makeig, 2004) for ERP analysis, and a bandpass filter of $0.1-20 \mathrm{~Hz}$ was applied. The data were segmented into epochs spanning $200 \mathrm{~ms}$ before the onset of button pressing to $1000 \mathrm{~ms}$ after. This epoching was performed separately for each participant and each type of operation response. Extended independent component analysis was conducted using the runica algorithm (Bell and Sejnowski, 1995) implemented in EEGLAB. The resultant EEG artifacts accounting for eye movement and muscle potentials were removed from the data. The ERP waveforms were obtained by averaging the data from the aforementioned epochs. For each averaged waveform, the 200 -ms period preceding stimulus onset was defined as the baseline. The P300 component was designated as the largest positive peak occurring between 250 and $600 \mathrm{~ms}$ after the release button was pressed. The baseline-to-peak P300 amplitudes were measured at Pz, the dominant electrode site. The P300 amplitude for the Pz electrodes for each type of operation response was used as the dependent variable for the repeated measures analysis of variance (ANOVA). The significance level was set at 5\%. 


\subsection{Results and Discussion}

Figure 5 shows the average Likert scale score, which indicates the degree of subjective discrepancy, for each type of operation response. Relative to the combined presentation of the auditory and tactile stimuli and the auditory stimulus alone, the subjective discrepancy scores for the tactile stimulus alone and no stimuli were higher; hence, the subjective sense of discrepancy was high when the camera operation was not followed by sound. A repeated measures one-factor ANOVA was performed with the type of operation response as the independent variable and the score for the sense of discrepancy as the dependent variable. The type of operation response did not significantly affect the discrepancy score. Figure 6 shows the average P300 amplitude for each type of operation response, with the accompanying error bars representing the standard error. Relative to the combined presentation of the audio and tactile stimuli, all other operation response types were associated with higher average P300 amplitudes. Performing a repeated measures one-factor ANOVA with the type of operation response as the independent variable and P300 amplitudes as the dependent variable, we found a significant main effect of the type of operation response $(F=6.840, p=.003)$. Multiple comparisons revealed that the P300 amplitudes evoked by the tactile stimulus alone and no stimuli were significantly larger than those evoked by the combined auditory and tactile stimuli.

In Experiment 1, we investigated the applicability of P300 as a quantitative evaluation method to measure the sense of discrepancy of the operation response by operating the real single-lens camera. For low-frequency stimulus, it is known that P300 amplitude increases with attention to the stimulus (Polich, 2007). Among the three operation responses presented at low-frequency, tactile only, and no stimuli significantly increased the P300 amplitude more than the high-frequency combined auditory and tactile stimuli. The result suggests that the associations of the camera operation response with the subjective sense of discrepancy and the P300 amplitude showed the same tendency. In the research using a single-lens camera (Yanagisawa and Miyazaki, 2019), the participant asked to press the release button and evaluate the shutter sound, which is the operation response. The research showed that the participant expected the shutter sound from the camera's body and that an expectation effect on the subjective evaluation occurred when an unexpected shutter sound was heard. Previous research (Mars et al., 2008) reported that unexpected events draw attention and elevate the P300 amplitude, which can thus be used as an indicator of surprise. Our previous study showed that subjective surprise increased and P300 amplitude increased when a percussion instrument was struck and sounded differently than predicted (Yanagisawa et al., 2019). In other words, the sense of discrepancy occurred when the response to the instrument manipulation was different from what was predicted, which was consistent with the current findings. The results of this study indicate that when the operation response to the pressing of the release button of a camera differed from that associated with the typical operation of analog cameras, attention was directed to the operation response, and the P300 amplitude increased.

The results of this study suggest that the P300 amplitude could be used to evaluate the sense of discrepancy of the operation response of a product, such as the single-lens camera used in this study, if the product has some expectations of the operation response based on experience and social conventions. The application of the proposed method to product design is explained using a mirrorless camera as an example. When adding a digital sound and vibration to a mirrorless single-lens camera that does not generate sound and vibration, we could perform a P300 evaluation experiment using multiple prototypes as operation response. The prototype with the smallest P300 amplitude to the operation response could be used as an operation response with less discrepancy.

Experiment 1 had some limitations. First, the sample size was small and biased in terms of the age range. Since Experiment 1 used a within-participant factorial design, the individual difference had an insignificant effect between conditions. However, we believe it is necessary to increase the number of participants and various ages. Second, the results of the subjective indices in this study showed a tendency for the subjective sense of discrepancy to increase due to the lack of sound in the operational response, but the difference was not statistically significant. Third, digital sound and vibration were added to the mirrorless single-lens camera because it is easy to control experimentally. However, we believe that comparison of the results using a conventional single-lens camera that produces shutter sound and vibration will be necessary. Furthermore, verifying the proposed method by using various products and operational responses in the future would be necessary. 


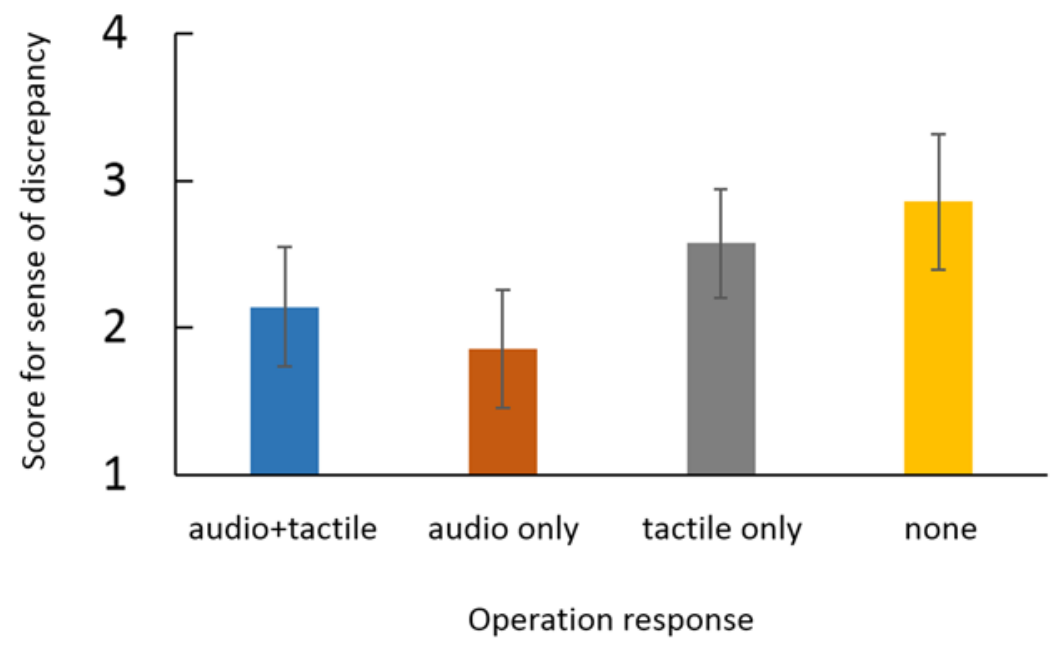

Fig. 5 Subjectively reported scores for sense of discrepancy for each type of operation response ( $\mathrm{n}=7$ ). The error bars represent the standard error.

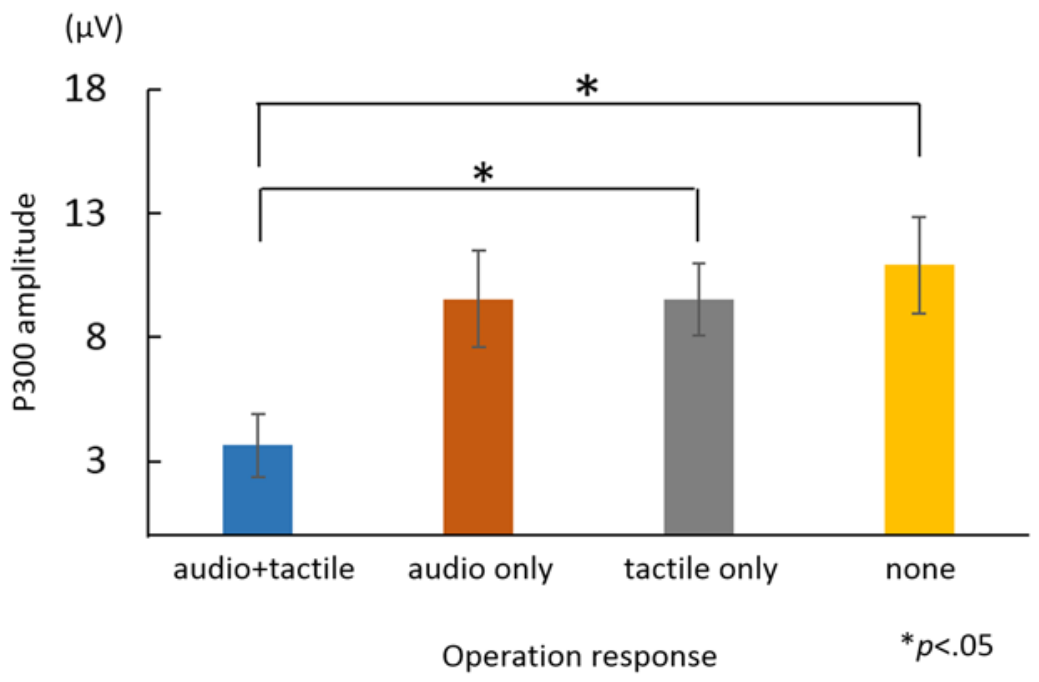

Fig. 6 P300 amplitudes for each type of operation response $(n=7)$. The error bars represent the standard error.

\section{Experiment 2: Evaluation of time transition of sense of discrepancy of operation response using P300}

In Experiment 2, we examined whether the P300 can be used to quantitatively evaluate the habituation of the sense of discrepancy of product operation responses using pure tone sounds that are easy to get used to and a blackboard scratching sound that is difficult to get used to as the low-frequency operation responses to mouse click operations in the oddball paradigm (Polich, 2007). We investigated whether the attenuation of the subjectively-rated distractibility and the P300 amplitude would show the same trend. We used mouse click operations so that the subjects do not get tired during continuous operation in Experiment 2.

\subsection{Methods}

\subsubsection{Participants}

The participants were six right-handed volunteers (five men; age range, 21-27 years) who had neither brain-related disorders nor any other disease. 


\subsubsection{Apparatus and stimuli}

The experimental setup is shown in Fig. 7. A display and speakers were set up in front of the participants. The participants sat on a chair and operated a mouse with their right hand on a pedestal set up on the right side. When the participants pressed the mouse button, an auditory stimulus was played over the speaker as a response. The input by the mouse click and the following auditory stimulus presentation were controlled using the stimulus presentation control software (Presentation, Neurobehavioral Systems, Inc., California, U.S.A.). A $250 \mathrm{~Hz}$ tone sound was presented as a high-frequency stimulus (duration, $120 \mathrm{~ms}$ ), a $420 \mathrm{~Hz}$ tone sound was presented as a low-frequency stimulus that was easy to get used to (duration, $120 \mathrm{~ms}$ ), and a blackboard scratching sound was presented as a low-frequency stimulus that was difficult to get used to (duration, $300 \mathrm{~ms}$ ).

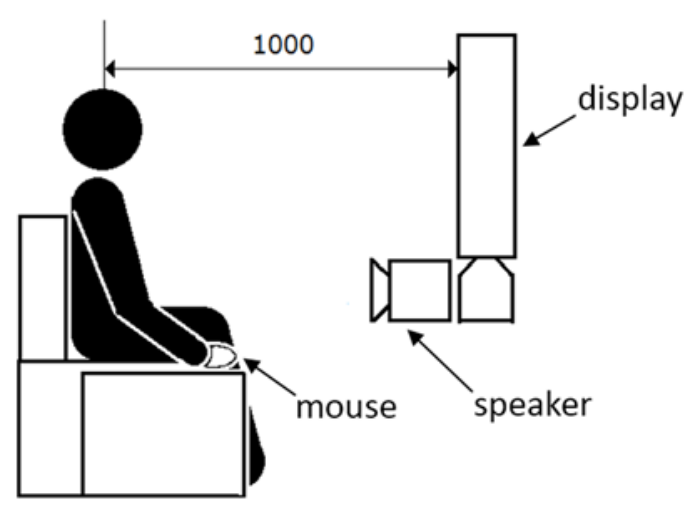

Fig. 7 Outline of experimental setup (unit: $\mathrm{mm}$ ).

\subsubsection{Procedure}

After receiving an overview of the experiment, the participants sat on a comfortable chair and were attached to the EEG electrodes. Participants were then asked to click the mouse button. To prevent drowsiness caused by monotonous mouse clicks, we asked the participants to perform the following task:

(1) When the task started, a white horizontal bar, a red cursor, and an orange target were displayed (Fig. 8). Orange targets were displayed at random locations on the horizontal bar.

(2) The participants were to use the mouse to move the cursor. When the participant moved the cursor to the center of the target, the cursor stopped, and the participant clicked the mouse button.

(3) The auditory stimulus was presented as an operation response to the click of the mouse button.

The participants performed 720 trials of this task. The inter-trial interval was 1 to 2 seconds. The position of the target changed with each trial, and the participants again manipulated the position of the cursor with the mouse. We divided the experiment into three blocks of 240 trials to investigate the habituation of the sense of discrepancy of the operation response (Block 1: 1-240 trial, Block 2: 241-480 trial, Block 3: 481-720 trial). In each block, the 250-Hz tone sound (160 trials), 420-Hz tone sound (40 trials), and blackboard scratching sound (40 trials) were presented in random order. The participants were asked to rate the distractibility to the operation response on a 4-point Likert scale $(1=$ "less distractable", 4 = "more distractable") at the end of each block. 


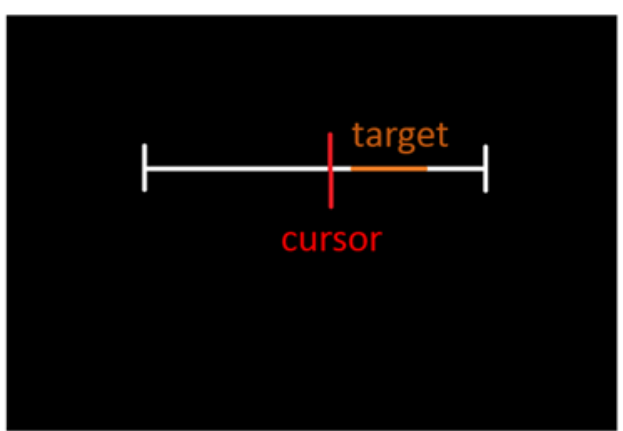

Fig. 8 Example of experimental task.

\subsubsection{EEG recordings and EEG data analysis}

EEGs were recorded with a digital electroencephalograph (eego sports, ANT Neuro, Hengelo, Netherlands). EEG was measured from 31 active electrodes on the scalp (Fp1/Fp2, Fpz, F7/F8, F3/F4, Fz, FC5/FC6, FC1/FC2, M1/M2, T7/ T8, C3/C4, Cz, CP5/CP6, CP1/CP2, P7/P8, P3/P4, Pz, POz, and O1/O2) with an electrode placed on the top of the nose $(\mathrm{Nz})$ as a reference according to the international 10-20 system (sampling frequency $500 \mathrm{~Hz}$, time constant $3 \mathrm{~s}$ ). The P300 amplitudes for the high-frequency stimulus $(250 \mathrm{~Hz}$ tone sound) and the two low-frequency stimuli (420 $\mathrm{Hz}$ tone sound and blackboard scratching sound) were calculated for each block. The P300 amplitude was calculated in the same way as in Experiment 1 (Section 2.1.5). The P300 amplitude for the Pz electrodes for each type of operation response was used as the dependent variable for the repeated measures ANOVA. The significance level was set at 5\%.

\subsection{Results and Discussion}

Figure 9 shows the temporal transition of the average Likert scale score, which indicates the subjective distractibility to the operation response. The subjective distractibility for the high-frequency stimulus (250 Hz tone) was maintained at a low level. In all blocks, the blackboard scratching sound was rated as more likely to attract attention than the $420 \mathrm{~Hz}$ tone sound. For low-frequency stimuli (blackboard scratching sound and $420 \mathrm{~Hz}$ tone), the rating of distractibility decreased throughout the block. To clarify the differences in the temporal transition between the low-frequency stimuli, a repeated measures two-factor ANOVA was performed with the type of operation response (blackboard scratching sound and $420 \mathrm{~Hz}$ tone) and number of blocks as independent variables and the score for distractibility as the dependent variable. We found a significant main effect of the type of operation response $(F=27.405, p=.000)$ and number of blocks $(F=4.378, p=.020)$. This result suggests that the blackboard scratching sound attracted more attention than the $420 \mathrm{~Hz}$ tone sound, meaning it was perceived as a discrepancy. In addition, the distractibility decreased with time for both types of operation response, indicating a decrease in discrepancy. Figure 10 shows the time transition of the P300 amplitude for each operation response. Similar to the subjective evaluation, the P300 amplitude for the $250 \mathrm{~Hz}$ tone was maintained at a low level. The P300 amplitude of the blackboard scratching sound was higher than that of the $420 \mathrm{~Hz}$ tone for all blocks. Performing a repeated measures two-factor ANOVA with the type of operation response (blackboard scratching sound and $420 \mathrm{~Hz}$ tone) and number of blocks as independent variables and P300 amplitudes as the dependent variable, we found significant main effects for the type of operation response $(F=11.836, p=.018)$ and number of blocks $(F=4.580, p=.039)$, and a significant interaction for the type of operation response and number of blocks $(F=4.433, p=.042)$. The $\mathrm{P} 300$ amplitude for the $420 \mathrm{~Hz}$ tone sound remained at the same level over time, while that for the blackboard scratching sound was higher than that for the $420 \mathrm{~Hz}$ tone sound, and was attenuated over time.

In Experiment 2, the degree of subjective distractibility and the P300 amplitude showed similar habituation in the operation response to mouse clicks. These results provide evidence that the P300 amplitude can evaluate the habituation of the sense of discrepancy. Similar to the subjective evaluation, the P300 amplitude for the blackboard scratching sound was larger than the $420 \mathrm{~Hz}$ tone sound at any time. In addition, the P300 amplitude declined over time for both types of operation responses. Previous studies have shown that the P300 amplitude decreased with repeated presentation using simple and passive stimulus (Polich, 1989; Lew and Polich, 1993; Polich and McIsaac, 1994; Romero and Polich, 1996; Ravden and Polich, 1998; 1999; Pan et al., 2000; Wintink et al., 2001; Kececi et al., 2006; 
Fonken et al., 2019; Barry et al., 2020; Ueda et al., 2020). Those findings are consistent with the current findings of the attenuation of the P300 amplitude to the operation response during repeated product manipulation. The application of the proposed method to design is explained below using a mouse click as an example. To add a digital click sound to a mouse click without any discrepancy, we could conduct continuous operation experiments using multiple prototypes as the operation response. The prototype, which had a fast decay of P300 amplitude for the operation response, could be used as an easy to get used to operation response.

Experiment 2 is also limited by the small number of samples and an age bias, as in Experiment 1 . However, since Experiment 2 was conducted in a within-participant factorial design, we consider the influence of individual differences on each condition to be small. Nevertheless, it is necessary to increase the number of participants in the future. It was hypothesized that the $420 \mathrm{~Hz}$ sound would be habituated to more quickly than the blackboard scratching sound and that the P300 amplitude would decay faster over time. However, the P300 amplitude for the 420 Hz sound was at a low level from the beginning and remained at that level thereafter. This result may be because the $420 \mathrm{~Hz}$ sound had little sense of discrepancy with respect to the operation response of the mouse click. Further research will be needed using manipulation reactions with varying degrees of unfamiliarity in various products.

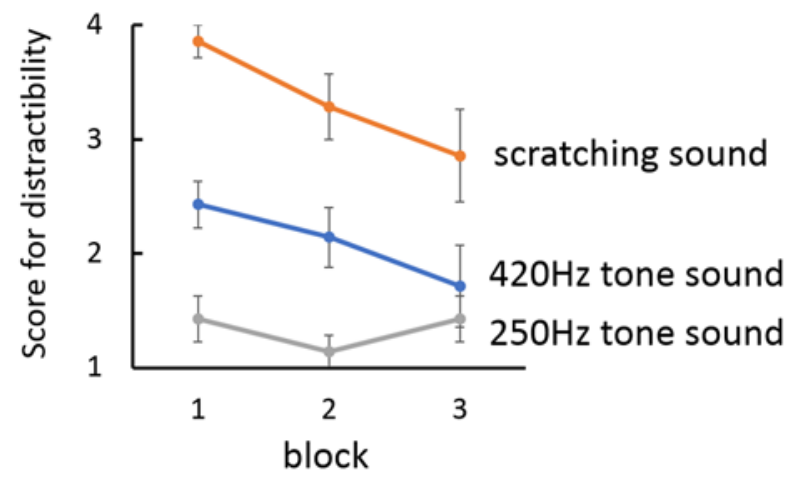

Fig. 9 Subjectively reported scores for distractibility for each type of operation response $(n=6)$.

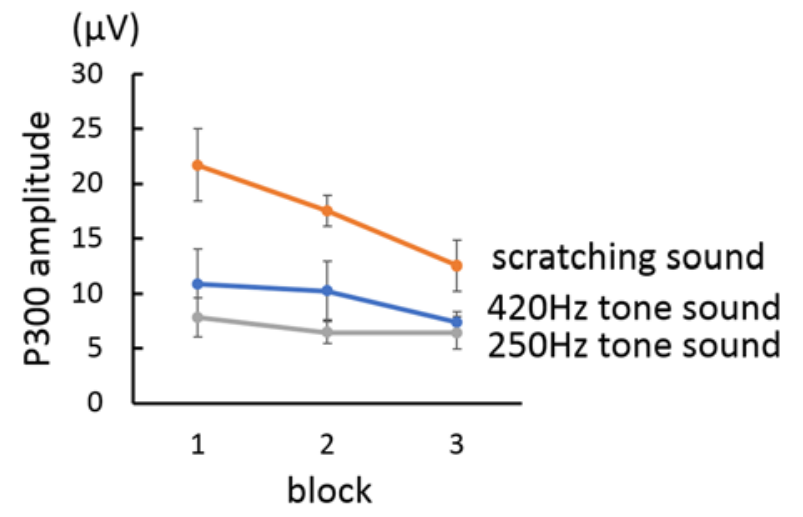

Fig. 10 P300 amplitude for each type of operation response $(n=6)$.

\section{Conclusion}

This study aimed to develop a method to quantitatively evaluate the user's sense of discrepancy to the production operation response and the habituation to the response. We examined the prospect of an ERP component (i.e., the P300) considered to reflect attention to a stimulus, to evaluate the sense of discrepancy to the product response to the user's action. In Experiment 1, using subjective evaluation and P300 to investigate the sense of discrepancy due to the lack of operation response (sound and vibration) to the shutter operation of the mirrorless single-lens camera, it was confirmed that the P300 amplitude corresponded to the degree of the subjective sense of discrepancy. Moreover, in Experiment 2 , modulating the operation response to the mouse click operation, the attenuation of the P300 amplitude to the operation response during the repeated operation was consistent with the subjective tendency of the distractibility. Our results 
showed that the P300 amplitude could help determine the sense of discrepancy of the operation response and the habituation to the response. We believe that our proposed method of evaluating the sense of discrepancy of the operation response using P300 amplitude will help design or improve the operation response. When designing an operation response, by implementing several prototypes as the operation response in a product and evaluating the P300 amplitude for each operation response, it is possible to select a prototype that is less sense of discrepancy and does not cause a persistent sense of discrepancy. The proposed method could also be used to identify the optimal sensory modality of the operation response for a product. In future studies, it is necessary to conduct experiments on various products' operation responses to improve the results' robustness further.

\section{Acknowledgements}

This research was carried out through a collaborative research project with the Sony Corporation. We would like to express our gratitude to Dr. Kazuko Yamagishi of Sony Global Manufacturing \& Operations Corporation and those who cooperated. This work was supported by KAKEN grant number $18 \mathrm{H} 03318$ from the Japan Society for the Promotion of Science.

\section{References}

Barry, R.J., Steiner, G.Z., De Blasio, F.M., Fogarty, J.S., Karamacoska, D., and MacDonald, B., Components in the P300: Don't forget the Novelty P3! Psychophysiology, Vol. 57, No. 7 (2020), e13371.

Bell, A.J., and Sejnowski, T.J., An information-maximization approach to blind separation and blind deconvolution. Neural computation, Vol. 7, No. 6 (1995), 1129-1159. doi: 10.1162/neco.1995.7.6.1129.

Delorme, A., and Makeig, S., EEGLAB: an open source toolbox for analysis of single-trial EEG dynamics including independent component analysis. Journal of Neuroscience Methods, Vol. 134, No. 1 (2004), 9-21. doi: 10.1016/j.jneumeth.2003.10.009.

Fonken, Y.M., Kam, J.W., and Knight, R.T., A differential role for human hippocampus in novelty and contextual processing: Implications for P300. Psychophysiology, Vol. 57, No. 2 (2019), e13400. doi: 10.1111/psyp.13400.

Gray, H.M., Ambady, N., Lowenthal, W.T., and Deldin, P., P300 as an index of attention to self-relevant stimuli. Journal of Experimental Social Psychology, Vol. 40, No. 2 (2004), 216-224. doi: 10.1016/S0022-1031(03)00092-1.

Kececi, H., Degirmenci, Y., and Atakay, S., Habituation and dishabituation of P300. Cognitive and Behavioral Neurology, Vol. 19, No. 3 (2006), 130-134. doi: 10.1097/01.wnn.0000213911.80019.c1.

Klem, G.H., Luders, H.O., Jasper, H.H., and Elger, C., The ten-twenty electrode system of the International Federation. The International Federation of Clinical Neurophysiology. Electroencephalography and Clinical Neurophysiology, Vol. 52, (1999), 3-6.

Lew, G.S., and Polich, J., P300, habituation, and response mode. Physiology \& Behavior, Vol. 53, No. 1 (1993), 111-117. doi: 10.1016/0031-9384(93)90018-B.

Mars, R.B., Debener, S., Gladwin, T.E., Harrison, L.M., Haggard, P., Rothwell, J.C., et al., Trial-by-Trial Fluctuations in the Event-Related Electroencephalogram Reflect Dynamic Changes in the Degree of Surprise. Journal of Neuroscience, Vol. 28, No. 47 (2008), 12539-12545. doi: 10.1523/JNEUROSCI.2925-08.2008.

Okubo, M., Suzuki, H., and Nicholls, M.E., A Japanese version of the FLANDERS handedness questionnaire. Shinrigaku Kenkyu, Vol. 85, No. 5 (2014), 474-481. doi: 10.4992/jjpsy.85.13235.

Pan, J., Takeshita, T., and Morimoto, K., P300 habituation from auditory single-stimulus and oddball paradigms. International Journal of Psychophysiology, Vol. 37, No. 2 (2000), 149-153. doi: 10.1016/s0167-8760(00)00086-6.

Polich, J., Habituation of P300 from auditory-stimuli. Psychobiology, Vol. 17, No. 1 (1989), 19-28. doi: 10.3758/BF03337813.

Polich, J., Updating p300: An integrative theory of P3a and P3b. Clinical Neurophysiology, Vol. 118, No. 10 (2007), 2128-2148. doi: 10.1016/j.clinph.2007.04.019.

Polich, J., and McIsaac, H.K., Comparison of auditory P300 habituation from active and passive conditions. 
International Journal of Psychophysiology, Vol. 17, No. 1 (1994), 25-34. doi: 10.1016/0167-8760(94)90052-3. Ravden, D., and Polich, J., Habituation of P300 from visual stimuli. International Journal of Psychophysiology, Vol. 30, No. 3 (1998), 359-365. doi: 10.1016/s0167-8760(98)00039-7.

Ravden, D., and Polich, J., On P300 measurement stability: habituation, intra-trial block variation, and ultradian rhythms. Biological Psychology, Vol. 51, No. 1 (1999), 59-76. doi: 10.1016/s0301-0511(99)00015-0.

Romero, R., and Polich, J., P3(00) habituation from auditory and visual stimuli. Physiology \& Behavior, Vol. 59, No. 3 (1996), 517-522. doi: 10.1016/0031-9384(95)02099-3.

Ueda, K., Sekoguchi, T., and Yanagisawa, H., How predictability affects habituation to novelty? bioRxiv, (2020), doi: 10.1101/2020.07.24.219253.

Wintink, A.J., Segalowitz, S.J., and Cudmore, L.J., Task complexity and habituation effects on frontal P300 topography. Brain and Cognition, Vol. 46, No. 1-2 (2001), 307-311. doi: 10.1016/s0278-2626(01)80090-7.

Yanagisawa, H., Kawamata, O., and Ueda, K., Modeling emotions associated with novelty at variable uncertainty levels: a Bayesian approach. Frontiers in Computational Neuroscience, Vol. 13, (2019), 2. doi: 10.3389/fncom.2019.00002.

Yanagisawa, H., and Miyazaki, C., A methodology for extracting expectation effect in user-product interactions for multisensory experience design. Journal of Advanced Mechanical Design Systems and Manufacturing, Vol. 13, No. 1 (2019), doi: 10.1299/jamdsm.2019jamdsm0013. 